Portland State University

PDXScholar

$11-15-1980$

\title{
Dielectric Saturation in Dipolar Fluids. I. The Single- Molecule Distribution Function
}

John D. Ramshaw

Portland State University, jdramshaw@yahoo.com

Follow this and additional works at: https://pdxscholar.library.pdx.edu/phy_fac

Part of the Physics Commons

Let us know how access to this document benefits you.

\section{Citation Details}

J.D. Ramshaw, "Dielectric saturation in dipolar fluids. I. The single-molecule distribution function," J. Chem. Phys. 73, 3695 (1980)

This Article is brought to you for free and open access. It has been accepted for inclusion in Physics Faculty Publications and Presentations by an authorized administrator of PDXScholar. Please contact us if we can make this document more accessible: pdxscholar@pdx.edu. 


\title{
Dielectric saturation in dipolar fluids. I. The single-molecule distribution function ${ }^{\text {a) }}$
}

\author{
John D. Ramshaw \\ Theoretical Division, University of California, Los Alamos Scientific Laboratory, Los Alamos, New \\ Mexico 87545 \\ (Received 27 February 1980; accepted 16 July 1980)
}

\begin{abstract}
The functional-derivative formalism is used to investigate the form of the equilibrium single-molecule distribution function $n(1)$ in a finite fluid system of rigid polar molecules subjected to a strong external electric field that varies slowly with position. The investigation is based on the assumption that the long-range asymptotic behavior of the direct correlation function is independent of the external field, and is hence the same as in the unperturbed fluid. This assumption implies that $n(1)$ has the form of a single-molecule Boltzmann factor in which the interaction energy is that of a deformable quasidipole with the local Lorentz electric field $\mathbf{E}_{L}\left(\mathbf{r}_{L}\right)$. If the chemical potential $\mu$ is held fixed, $n(1)$ is a local function of $\mathbf{E}_{L}\left(\mathbf{r}_{\downarrow}\right)$. In a closed system, however, the number of molecules $N$ is held fixed and $\mu$ varies with the field. Consequently, $n(1)$ is an inherently nonlocal functional of $\mathbf{E}_{L}(\mathbf{r})$, except in the linear approximation where local behavior is regained. The implications of these results for the polarization and higher angular moments of $n(1)$ will be explored in a subsequent article.
\end{abstract}

\section{INTRODUCTION}

This article and its sequel are part of a continuing study of dielectric behavior and pair correlations in fluids composed of rigid (unpolarizable) polar molecules. Previous work in this study ${ }^{1-6}$ has been concerned with linear dielectric polarization and its relation to pair cor relations in the unperturbed fluid. A primary objective of this work has been to clarify the conditions under which there is a local proportionality between the polar ization $\mathbf{P}(\mathbf{r})$ and the Maxwell electric field $\mathbf{E}(\mathbf{r})$, so that the dielectric constant $\epsilon$ exists and is a property of the sample material. It was found that the direct correlation function $c(12)$ is of fundamental importance to this question, and that its asymptotic behavior at long range is especially crucial. ${ }^{2}$ The importance of $c(12)$ in this context is due to its interpretation in terms of an inverse response kernel, an interpretation provided by the functional-derivative approach to the theory of fluids. ${ }^{7,8}$ The known asymptotic behavior of $c(12)$ [together with the assumption that $c(12)$ depends only on relative positions and orientations at short range] is sufficient to establish the existence of $\epsilon$, provided that the external electric field varies slowly with position in a molecular sense. ${ }^{4,5}$ When the latter condition is violated, the relation between $\mathbf{P}(\mathbf{r})$ and $\mathbf{E}(\mathbf{r})$ assumes a nonlocal convolution form. ${ }^{6}$ The convolution kernel is short ranged, however, so that $\mathbf{P}(\mathbf{r})$ still depends only on the values of $\mathbf{E}$ in the molecular neighborhood of the point $\mathbf{r}$.

Our purpose here is to study dielectric saturation in dipolar fluids from a similar point of view. By the term "dielectric saturation" we refer in a general way to the nonlinear response of the system to a strong static external electric field. The polarization $\mathbf{P}(\mathbf{r})$ is an important aspect of this response, but it is by no means the only response quantity of interest. Considerably more information is obtained, at no extra cost, by considering the single-molecule distribution function $n(1)$,

\footnotetext{
a) Work performed in part under the auspices of the United States Department of Energy.
}

from which $\mathbf{P}(\mathbf{r})$ and many other observable properties of the system may be obtained by quadrature. The pres ent article is ther efore devoted to an analysis of $n(1)$. Attention is restricted to external fields that vary slowly with position in a molecular sense.

In the present context, it becomes necessary to use the finite-field form of the functional-derivative relations, instead of the zero-field form that sufficed for the linear case. The direct correlation function and its asymptotic behavior again assume a position of crucial importance. The development is based upon the fundamental assumption that the asymptotic form of $c(12)$ is independent of the field, and is ther efore the same as in zero field. The validity of this assumption is implied by the same sort of formal cluster-expansion procedures that are used to infer the asymptotic form of $c(12)$ in an unperturbed fluid. ${ }^{9}$ This assumption implies an expression for $n(1)$ in the form of a single-molecule Boltzmann factor, in which the interaction energy is that of a deformable (polarizable) quasidipole with the local Lorentz electric field $\mathbf{E}_{L}\left(\mathbf{r}_{1}\right)$. This new formal expression for $n(1)$ is valid to all orders in the field strength, and is the principal result of this article.

For technical reasons, the development is carried out in the grand canonical ensemble, in which the chemical potential $\mu$ replaces the number of molecules $N$ as a basic independent variable. At constant $\mu, n(1)$ is a local function of $\mathbf{E}_{L}\left(\mathbf{r}_{1}\right)$, but this is unfortunately not the case of interest. We are concerned with the behavior of a closed system, in which $N$ is held fixed and $\mu$ varies with the field. We must therefore eliminate $\mu$ in favor of $N$. However, the relation between $\mu$ and $N$ involves $\mathbf{E}_{L}(\mathbf{r})$ in an inherently nonlocal way, i. e., at fixed $N, \mu$ is a nonlocal functional of $\mathbf{E}_{L}(\mathbf{r})$. When $\mu$ is eliminated, therefore, $n(1)$ becomes an inherently nonlocal functional of $\mathbf{E}_{L}(\mathbf{r})$. The only exception to this behavior occurs in the linear approximation, in which local behavior is regained.

We emphasize that this nonlocal behavior is unrelated 
to, and quite different in character from, that which accompanies rapid spatial variations in the electric field. In the latter case, the nonlocal behavior occurs even in the linear regime, but it is confined to the molecular neighborhood of the point in question and it vanishes for slowly varying fields. In contrast, the present nonlocal behavior occurs when the field is slowly varying and includes contributions from the entire sample volume, but it is intrinsically nonlinear and vanishes in the linear approximation.

Our conclusions about the form and nonlocal behavior of $n(1)$ have interesting implications for the various observable quantities that can be expressed in terms of $n(1)$. A detailed discussion of these implications for the polarization $\mathbf{P}(\mathbf{r})$ and higher angular moments of $n(1)$ will be deferred to a subsequent article. Here we merely observe that the local number density $\rho\left(\mathbf{r}_{1}\right)=\int d \omega_{1} n(1)$ is also a nonlocal functional of $\mathbf{E}_{L}(\mathbf{r})$, except in the linear approximation where it is uniform. It is similarly clear that $\mathbf{P}\left(\mathbf{r}_{1}\right)=\int d \omega_{1} \mathrm{~m}_{1} n(1)$ is in general a nonlocal functional of $\mathbf{E}_{L}(\mathbf{r})$, contrary to intuitive expectations. In the linear regime, however, $\mathbf{P}(\mathbf{r})$ becomes locally proportional to $\mathbf{E}_{L}(\mathbf{r})$, in agreement with our earlier results for this case. $^{4,5}$

Most previous work on dielectric saturation in polar fluids has been concerned with the global response of the sample as a whole, rather than with the more general questions of local behavior which concern us here. Work of the former type has been reviewed by Kielich. ${ }^{10}$ Previous analyses of the single-molecule distribution in both $\mathbf{r}_{1}$ and $\omega_{1}$ appear to be limited to those of Nienhuis and Deutch ${ }^{11}$ (ND) and Hoye and Stell ${ }^{9}$ (HS). Although there are several points of contact between our work and that of HS, they focus for the most part on a different set of issues than we do. They are concerned with an infinite system rather than a finite closed system, and nonlocal effects consequently play no role in their development. Moreover, they are primarily interested in quantities other than $n(1)$, and obtain explicit results for the latter only in the weak-field and mean-field limits. ND, on the other hand, attempt to derive a general formal expression for $n(1)$ in a finite closed system, and it is of interest to contrast our result with theirs.

The result of the ND theory reduces, for slowly varying fields, to their Eq. (4.30) for $n(1)$ as an infinite series in $\mathbf{E}(\mathbf{r})$, the Maxwell electric field. (The appearance of the Maxwell field rather than the Lor entz field is not an essential difference; it merely reflects a dif fer ent convention in the definition of the dipole tensor.) The coefficients in this series involve complicated $n$ molecule correlation functions in the unperturbed fluid. Because of the infinite series and the reduction to zerofield correlations, the ND expression for $n(1)$ bears no appar ent resemblance to our closed-form expression. One may still inquire, however, whether the two expressions are somehow equivalent. The answer is that they are not, because the ND result for $n(1)$ is a purely local function of $\mathbf{E}\left(\mathbf{r}_{1}\right)$ [or, equivalently, of $\mathbf{E}_{L}\left(\mathbf{r}_{1}\right)$ ], whereas our result is inherently nonlocal.

We believe that this discrepancy is due to the fact $^{12}$ that ND neglect the finite-volume correction terms that appear in the distribution functions of a finite system in the canonical ensemble. These terms are not negligible in the present context, ${ }^{13}$ and their omission leads to serious error. It seems likely, however, that a derivation similar in structure to that of ND could be performed in the grand canonical ensemble, and that the resulting expression for $n(1)$ would be similar in form to that of ND. Such a result would exhibit the same kind of nonlocal behavior that we have found here, because the zero-field correlation functions would then depend implicitly on $\mu$, which is a nonlocal functional of $\mathbf{E}(\mathbf{r})$. Even if this could be done, however, the expression for $n$ (1) derived herein has the advantage of being much simpler in structure, and it has an appealing intuitive interpretation in terms of a deformable quasidipole that is lacking in a series solution of the ND type. Thus, the present result is likely to be a more fruitful starting point for approximations: It requires only that one as sume physically reasonable behavior for the quasidipole, rather than for an infinite set of many-particle correlation functions.

\section{THE SINGLE-MOLECULE DISTRIBUTION FUNCTION}

We consider a system composed of $N$ identical rigid polar molecules (of arbitrary symmetry) confined to a finite volume $V$ (of arbitrary shape) at absolute temperature $T$. The mean number density $N / V$ is denoted by $\bar{\rho}$. The position and orientation of molecule $k$ are denoted by $\mathbf{r}_{k}$ and $\omega_{k}$, respectively, and are collectively represented by the shorthand notation $(k)$. The angular measure $\int d \omega_{1}$ is denoted by $\Omega$. If $\omega_{1}$ is specified by the Euler angles, ${ }^{14}$ then $\Omega=8 \pi^{2}$. The dipole moment of molecule $k$ is denoted by $\mathbf{m}_{k}$. Since the molecules are rigid, $\mathbf{m}_{k}$ depends only on $\boldsymbol{\omega}_{k}$, and its magnitude is a constant $m_{0}$.

The system is imagined to be suspended in vacuum in the presence of a strong external electric field $\mathbf{E}_{0}(\mathbf{r})$. The latter is assumed to vary slowly (in a molecular sense) with the position $r$, but is otherwise arbitrary. The interaction energy of the sample with the field is $\Sigma_{k} \phi_{0}(k)$, where

$$
\phi_{0}(1)=-\mathbf{m}_{1} \cdot \mathbf{E}_{0}\left(\mathbf{r}_{1}\right) .
$$

The properties of the sample in the presence of the external field may be considered as functionals of the single-molecule function $\phi_{0}(1)$, or alternatively of the function $\gamma_{0}(1)=-\beta \phi_{0}(1)$, where $\beta=(k T)^{-1}$ and $k$ is Boltz mann's constant. It is therefore not surprising that the calculus of functionals and functional derivatives will prove useful.

A convenient summary of the basic functional-derivative relations has been given by Rushbrooke. ${ }^{8}$ Although these relations are usually derived for spherical molecules interacting via short-range forces, they are also valid in the present context if certain reinterpretations are made. ${ }^{4}$ Specifically, it is necessary to interpret the notation $(k)$ in the generalized sense $\left(\mathbf{r}_{k}, \omega_{k}\right)$, to interpret $\rho$ as $N /(V \Omega)$, and to identify the spatial integration domain with the finite volume $V$.

The functional-derivative relations in their usual 
for $m^{7,8}$ are valid in the grand canonical ensemble. The corresponding relations in the canonical ensemble are somewhat different. ${ }^{13}$ The differences arise from the presence of a finite-volume correction term in the canonical total correlation function, and are related to the fact that canonical variations occur at constant $N$, while grand canonical variations occur at constant chemical potential $\mu$. [Throughout this article, $\mu$ refers to the total chemical potential of the system including that as sociated with the field, i.e., $\mu=-k T(\partial \ln Q / \partial N)_{V, T}$, where $Q$ is the canonical partition function in the presence of the external field.] The principal formal difference is that $\phi_{0}(1)$ is replaced by $\phi_{0}(1)-\mu$ in the canonical ensemble. In the linear approximation this difference vanishes, because $\mu$ contains no term linear in $\phi_{0}(1)$. The grand canonical relations may then be applied in the canonical ensemble, as was done in our previous work on linear dielectric polarization. ${ }^{4,5}$ In the present context, however, the field strength is arbitrary and the canonical and grand canonical formulations are no longer interchangeable. We must therefore select either one or the other, and consistently adhere to it throughout the development.

Since we are concerned with a closed system, we would prefer to use the canonical ensemble. The development is simpler, however, in the grand canonical ensemble, and the quantities that naturally appear are more easily given physical interpretations. We shall ther efore use the grand canonical ensemble, in which the usual functional-derivative relations apply without modification. It is essential to remember that the functional derivatives are then taken at constant $\mu$ rather than at constant $N$, and that a finite change in $\phi_{0}(1)$ at constant $\mu$ will be accompanied by a finite change in $N$. At the end of the derivation, it will therefore be necessary to eliminate $\mu$ as a basic independent variable in favor of $N$. It is this elimination that will give rise to nonlocal behavior.

We now proceed to summarize the relevant functionalderivative relations. The quantity of central interest is the single-molecule distribution function $n(r, \omega)$, which is the ensemble average of the molecular quantity $\Sigma_{k} \delta\left(\mathbf{r}-\mathbf{r}_{k}\right) \delta\left(\omega-\omega_{k}\right)$ in the presence of the external field $\mathbf{E}_{0}(\mathbf{r})$. Consider the consequences of effecting an infinitesimal change $\delta \mathbf{E}_{0}(\mathbf{r})$ in $\mathbf{E}_{0}(\mathbf{r})$ at constant $\mu$. The cor responding changes $\delta \phi_{0}(1)$ and $\delta \gamma_{0}(1)$ to the functions $\phi_{0}(1)$ and $\gamma_{0}(1)$ follow trivially from Eq. (1). The resulting change $\delta n(1)$ to $n(1)$ is

$$
\delta n(1)=\int d(2) \frac{\delta n(1)}{\delta \gamma_{0}(2)} \delta \gamma_{0}(2)
$$

where the functional derivative $\delta n(1) / \delta \gamma_{0}(2)$ is given by

$$
\frac{\delta n(1)}{\delta \gamma_{0}(2)}=n(1) n(2) h(12)+n(1) \delta(12)
$$

and $h(12)$ is the total correlation function in the presence of the field. The inverse relation to $\mathrm{Eq}$. (2) is

$$
\delta \gamma_{0}(1)=\int d(2) \frac{\delta \gamma_{0}(1)}{\delta n(2)} \delta n(2),
$$

$$
\frac{\delta \gamma_{0}(1)}{\delta n(2)}=-c(12)+\frac{1}{n(1)} \delta(12),
$$

and $c(12)$ is the direct correlation function in the presence of the field. It follows from Eqs. (2) $-(5)$ that $h(12)$ and $c(12)$ are related by the generalized OrnsteinZernike equation

$$
h(12)=c(12)+\int d(3) n(3) c(13) h(32) .
$$

The fundamental importance of $c(12)$ in dielectric theory is due to Eqs. (4) and (5), which allow one to isolate the external electric field $\mathbf{E}_{0}(\mathbf{r})$. This facilitates the elimination of $\mathbf{E}_{0}(\mathbf{r})$ in favor of the Maxwell electric field $\mathbf{E}(\mathbf{r})$, or, preferably, the Lorentz electric field $\mathbf{E}_{L}(\mathbf{r})=\mathbf{E}(\mathbf{r})$ $+(4 \pi / 3) \mathbf{P}(\mathbf{r})$. The fact that this elimination effects a simplification is a consequence of the particularly simple asymptotic behavior of $c(12)$ at long range (large $\left.\left|\mathbf{r}_{1}-\mathbf{r}_{2}\right|\right)$.

The asymptotic behavior of $c(12)$ in unperturbed fluids has been examined in a number of independent studies, many of which were cited in Ref. 4 . In these studies it was found that $c(12)$ becomes asymptotic to $-\beta v(12)$ at long range, where $v(12)$ is the intermolecular pair potential. The asymptotic behavior of $c(12)$ in the presence of an external field has received much less attention. Fortunately, the formal cluster-expansion procedures from which one infers the asymptotic form of $c(12)$ in zero field are largely indifferent to the presence of an external field, so indifferent indeed that the asymptotic form of $c(12)$ is found to be unaffected by the field. ${ }^{9}$ It would be an exaggeration to say that this result has been rigorously established, for the convergence properties of the cluster-expansion procedures are largely unknown (especially for liquids!). In the present development, therefore, we shall incorporate this result as an assumption, but one whose validity appears very likely. We assume, then, that $c(12)$ becomes asymptotic to $-\beta v(12)$ for large $\left|\mathbf{r}_{1}-\mathbf{r}_{2}\right|$, even in the presence of an extermal field. In the present context, the dipole-dipole potential dominates $v(12)$ at long range. Our assumption may therefore be stated in the form

$$
c(12)=c_{0}(12)-\beta \theta(12),
$$

where $c_{0}(12)$ is a short-ranged function (i.e., one that decays to zero faster than $\left.\left|\mathbf{r}_{1}-\mathbf{r}_{2}\right|^{-3}\right)$ and $\theta(12)$ is the dipole-dipole potential with a spherical cutoff

$$
\theta(12)=-m_{1} \cdot \mathbf{T}_{5}\left(\mathbf{r}_{12}\right) \cdot \mathrm{m}_{2} .
$$

Here $\mathbf{r}_{12}=\mathbf{r}_{1}-\mathbf{r}_{2}, \mathbf{T}_{5}(\mathbf{r})=H(|\mathbf{r}|-\delta) \nabla \nabla|\mathbf{r}|^{-1}, H(x)$ is zero if $x<0$ and unity otherwise, and it is understood that the limit $\delta \rightarrow 0$ is ultimately to be taken.

Combining Eqs. (4), (5), and (7), we obtain

$$
\delta \gamma_{L}(1)=\int d(2)\left[-c_{0}(12)+\frac{1}{n(1)} \delta(12)\right] \delta n(2) \text {, }
$$

where

$$
\gamma_{L}(1)=\gamma_{0}(1)-\beta \int d(2) \theta(12) n(2)
$$




$$
\mathbf{P}\left(\mathbf{r}_{1}\right)=\int d \boldsymbol{\omega}_{1} \mathbf{m}_{1} n(1) .
$$

This relation, together with Eqs. (1) and (8), allows Eq. (10) to be rewritten in the form

$$
\gamma_{L}(1)=\beta m_{1} \cdot E_{L}\left(\mathbf{r}_{1}\right),
$$

where

$$
\mathbf{E}_{L}\left(\mathbf{r}_{1}\right)=\mathbf{E}_{0}\left(\mathbf{r}_{1}\right)+\int d \mathbf{r}_{2} \mathbf{T}_{6}\left(\mathbf{r}_{12}\right) \cdot \mathbf{P}\left(\mathbf{r}_{2}\right)
$$

is the Lorentz electric field. The appearance of the Lorentz field, rather than the Maxwell field or some other electric field, is a consequence of the spherical cutoff in $\theta(12)$.

Equation (9) can be formally inverted by introducing a short-range total correlation function $h_{0}(12)$ that is related to $c_{0}(12)$ by the Ornstein-Zernike equation, i.e.,

$$
h_{0}(12)=c_{0}(12)+\int d(3) n(3) c_{0}(13) h_{0}(32)
$$

We then obtain

$$
\delta n(1)=\int d(2)\left[n(1) n(2) h_{0}(12)+n(1) \delta(12)\right] \delta \gamma_{L}(2) .
$$

Since $c_{0}(12)$ is a short-ranged function, the same is true of $h_{0}(12)$. The integrals over $\mathbf{r}_{2}$ in Eqs. (9) and (15) may therefore be extended over all space instead of just over the volume $V$, provided that $\mathbf{r}_{1}$ is farther than the range of $c_{0}(12)$ or $h_{0}(12)$ from the surface of $V$. The integral over $\mathbf{r}_{3}$ in $\mathrm{Eq}$. (14) may similarly be extended over all space.

Up to this point we have regarded $n(1)$ and the various correlation functions as functionals of $\gamma_{0}(1)$. Because of Eq. (10), we can equally well regard these quantities as functionals of $\gamma_{L}(1)$. Henceforth, we adopt this latter viewpoint. Equation (15) then shows that

$$
\frac{\delta n(1)}{\delta \gamma_{L}(2)}=n(1) n(2) h_{0}(12)+n(1) \delta(12) \text {, }
$$

where the functional differentiation is performed at constant $\mu$, just as in Eqs. (3) and (5). Since $h_{0}(12)$ is a short-ranged function, it follows from Eq. (16) that at constant $\mu, n(1)$ is appreciably influenced by $\gamma_{L}(2)$, and hence by $\mathbf{E}_{L}\left(\mathbf{r}_{2}\right)$, only when $\mathbf{r}_{2}$ is near $\mathbf{r}_{1}$. [Here and below the relative terms "near" and "far" are used with reference to the range of $h_{0}(12)$, which is a length of molecular magnitude.] At constant $\mu$, ther efore, $n(1)$ is a local functional of $\mathbf{E}_{L}(\mathbf{r})$, by which is meant that it is essentially independent of the values that $\mathbf{E}_{L}(\mathbf{r})$ takes on at points far from $\mathbf{r}_{1}$. However, according to $\mathrm{Eq}$. (16), $h_{0}(12)$ may be obtained from $n(1)$ by functional differentiation, which is the continuous analog of partial differentiation and which clearly cannot introduce any new functional dependence that is not already present in $n(1)$. [If $f\left(x_{1}, x_{2}\right)$ is independent of $x_{2}$, so that $\partial f / \partial x_{2}=0$, then the partial derivatives of $f\left(x_{1}, x_{2}\right)$ are also independent of $x_{2}$.] Therefore, $h_{0}(12)$ is also a local functional of $\mathbf{E}_{L}(\mathbf{r})$ at constant $\mu$; it is essentially independent of the values that $\mathbf{E}_{L}(\mathbf{r})$ takes on far from $\mathbf{r}_{1}$ and $\mathbf{r}_{2}$.

The conclusion that $h_{0}(12)$ is a local functional of $\mathbf{E}_{L}(\mathbf{r})$ at constant $\mu$ is crucial to our development. It is there- fore perhaps worthwhile to observe that this conclusion can be reached by alternate routes. Perhaps the simplest is to notice that if the contrary were true, func tional integration of Eq. (15) would yield an $n(1)$ that depends on $\mathbf{E}_{L}(\mathbf{r})$ at points far from $\mathbf{r}_{1}$, which contradicts the already established fact that $n(1)$ is a local functional of $\mathbf{E}_{L}(\mathbf{r})$. A second alternate route involves considering the effect of a small change in $\mathbf{E}_{L}\left(\mathbf{r}_{3}\right)$, where $\mathbf{r}_{3}$ is far from $r_{1}$ but is otherwise arbitrary. This change will not affect $n(1)$, and it therefore will not affect the change in $n$ (1) produced by a small change in $\mathbf{E}_{L}\left(\mathbf{r}_{2}\right)$, where $\mathbf{r}_{2}$ is near $r_{1}$. The latter change in $n(1)$ is related to $h_{0}(12)$ at short range by Eq. (16); therefore, the change in $\mathbf{E}_{L}\left(\mathbf{r}_{3}\right)$ does not affect $h_{0}(12)$ at short range. However, it does not affect $h_{0}(12)$ at long range either, since $h_{0}(12)$ is then essentially zero regardless of the value of $\mathbf{E}_{L}\left(\mathbf{r}_{3}\right)$. Therefore, the change in $\mathbf{E}_{L}\left(\mathbf{r}_{3}\right)$ does not affect $h_{0}(12)$ at all, so that $h_{0}(12)$ can depend only on $\mathbf{E}_{L}(\mathbf{r})$ at points near $\mathbf{r}_{1}$.

We have shown that both $n(1)$ and $h_{0}(12)$ are local functionals of $\mathbf{E}_{L}(\mathbf{r})$ at constant $\mu$. A further reduction in their functional dependence on $\mathbf{E}_{L}(\mathbf{r})$ results from the fact that the external field $\mathbf{E}_{0}(\mathbf{r})$ varies slowly with $\mathbf{r}$ in a molecular sense. Since the system is a fluid, it is clear that $n(1)$ will vary slowly with $\mathbf{r}_{1}$ in the same sense. This in turn implies, via Eqs. (11) and (13), that $\mathbf{P}(\mathbf{r})$ and $\mathbf{E}_{L}(\mathbf{r})$ also vary slowly with $\mathbf{r}$. The values that $\mathbf{E}_{L}(\mathbf{r})$ takes on at points near $\mathbf{r}_{1}$ ther efore differ negligibly from $E_{L}\left(r_{1}\right)$, so that in effect $n(1)$ and $h_{0}(12)$ depend on $\mathbf{E}_{L}(\mathbf{r})$ only through the single value $\mathbf{E}_{L}\left(\mathbf{r}_{1}\right)$. We may therefore consider $n(1)$ and $h_{0}(12)$ to be simply functions of $\mathbf{E}_{L}\left(\mathbf{r}_{1}\right)$ rather than functionals, which represents a major simplification of the mathematical description. It is this simplification that makes it possible for the development to proceed.

Substitution of Eq. (12) into Eq. (15) yields

$\delta n(1)=\beta n(1) \int d(2)\left[n(2) h_{0}(12)+\delta(12)\right] \mathbf{m}_{2} \cdot \delta \mathbf{E}_{L}\left(\mathbf{r}_{2}\right)$

Since $h_{0}(12)$ is short ranged and both $n(2)$ and $\mathbf{E}_{L}\left(r_{2}\right)$ are slowly varying functions of $\mathbf{r}_{2}$, we may evaluate these functions at the point $\mathbf{r}_{2}=\mathbf{r}_{1}$ to obtain

$$
\delta n(1)=\beta n(1) \mathbf{m}_{1}^{*} \cdot \delta \mathbf{E}_{L}\left(\mathbf{r}_{1}\right)
$$

where

$$
\mathrm{m}_{1}^{*}=\mathrm{m}_{1}+\int d(2) n\left(\mathbf{r}_{1}, \omega_{2}\right) h_{0}(12) \mathrm{m}_{2} .
$$

As will become clear later, $m_{1}^{*}$ may be interpreted as an effective dipole moment of molecule 1 . Unlike the permanent moment $m_{1}$, the effective moment $\mathbf{m}_{1}^{*}$ depends on $\mathbf{E}_{L}\left(\mathbf{r}_{1}\right)$ and is therefore deformable or polarizable. Our conclusions about the functional dependence of $n(1)$ and $h_{0}(12)$ imply that $\mathbf{m}_{1}^{*}$ may be written as $\mathbf{m}_{1}^{*}\left(1, \mathbf{E}_{L}\left(\mathbf{r}_{1}\right)\right)$. Of course, $m_{1}^{*}$ also carries an implicit parametric dependence on $\mu$.

We now observe that for given values of $r_{1}, \omega_{1}$, and $\mu$, Eq. (18) is simply an ordinary differential equation for $n(1)$. [It is no longer a functional differential equation because it does not involve $E_{L}(r)$ at points other than $r$ $=\mathbf{r}_{1}$.] The solution of this equation is 


$$
n(1)=\Omega^{-1} \rho_{0}(\mu, T) \exp \left[\beta \int_{0}^{\mathbf{z}_{L}\left(\mathbf{r}_{1}\right)} \mathrm{m}_{1}^{*}(1, \mathbf{E}) \cdot d \mathbf{E}\right],
$$

where $\mathbf{E}$ is a dummy integration variable representing $\mathbf{E}_{L}\left(\mathbf{r}_{1}\right)$, and we have imposed the boundary condition that $n(1)=\Omega^{-1} \rho_{0}(\mu, T)$ when $\mathbf{E}_{L}(\mathbf{r})=0$. Here $\rho_{0}(\mu, T)$ is the unifor $m$ number density in the system when the chemical potential is $\mu$ in the absence of the field, which is deter mined by the equation of state of the unperturbed fluid. The integration in Eq. (20) is of course carried out at constant $\mu$. Equation (20) again shows that at fixed $\mu$, $n(1)$ is a purely local function of $\mathbf{E}_{L}\left(\mathbf{r}_{1}\right)$.

Equation (20) has an appealing intuitive interpretation, which is due to the fact ${ }^{15,16}$ that the interaction energy of an isolated deformable dipole $m$ with an external electric field $\mathbf{E}$ is just $-\int \mathbf{m} \cdot d \mathbf{E}$. Ther efore, $n(1)$ is given simply by a single-molecule Boltzmann factor in which the interaction energy that appears is just that of the deformable effective moment $\mathbf{m}_{1}^{*}$ with the field $\mathbf{E}_{L}\left(\mathbf{r}_{1}\right)$. Us ing the language of field theory, we may say that $\mathbf{m}_{1}^{*}$ is a renormalized dipole moment; it is the dipole moment of a deformable quasiparticle that effectively interacts only with the field $\mathbf{E}_{L}\left(\mathbf{r}_{1}\right)$.

Equation (20) expresses $n(1)$ as a function of the independent variables $(\mu, V, T)$ and $\mathbf{E}_{L}(\mathbf{r})$. The dependence of $n(1)$ on $\mu$ occurs through both $\rho_{0}(\mu, T)$ and $\mathbf{m}_{1}^{*}$. Since we are concerned with a closed system, we wish to eliminate $\mu$ in favor of $N$, so that we may evaluate $n(1)$ for given $(N, V, T)$ and $\mathbf{E}_{L}(\mathbf{r})$. It is convenient to let

$$
\mathcal{E}(1, \mu)=\exp \left[\beta \int_{0}^{\mathbf{s}_{L}\left(\mathbf{r}_{1}\right)} \mathbf{m}_{1}^{*}(1, \mathbf{E}) \cdot d \mathbf{E}\right],
$$

whose dependence on $\mu$ is now explicitly indicated by the notation. In terms of $\mathcal{E}$, we have that $n(1)=\Omega^{-1} \rho_{0}(\mu$, $T) \mathscr{E}(1, \mu)$. The number of molecules $N$ in the system in the presence of the field is simply $N=\int d(1) n(1)$, i. e.,

$$
N=N\left(\mu,\left[\mathbf{E}_{L}(\mathbf{r})\right]\right)=\Omega^{-1} \rho_{0}(\mu, T) \int d(1) \mathcal{E}(1, \mu),
$$

which is the relation between $N$ and $\mu$. Equations (21) and (22) show that for given $\mu, N$ is a nonlocal functional of $\mathbf{E}_{L}(\mathbf{r})$. Conversely, for given $N, \mu$ is clearly a nonlocal functional of $\mathbf{E}_{L}(\mathbf{r})$, which may be written as $\mu(N$, $\left.\left[\mathbf{E}_{L}(\mathbf{r})\right]\right)$. This functional is determined implicitly by $\mathrm{Eq}$. (22).

Combining Eqs. (20) and (22), we find that $n(1)$ can be written as

$$
n(1)=\frac{N \mathcal{E}(1, \mu)}{\int d(1) \mathcal{E}(1, \mu)},
$$

which obviously has the correct normalization. In or der to express $n(1)$ as a function of the independent variables $(N, V, T)$ and $\mathbf{E}_{L}(\mathbf{r})$, it is merely necessary to substitute the functional relation $\mu\left(N,\left[\mathbf{E}_{L}(\mathbf{r})\right]\right)$ into $\mathrm{Eq} .(23)$. The resulting $n(1)$ is clearly an inherently nonlocal functional of $\mathbf{E}_{L}(\mathbf{r})$. Even if $\mathbf{m}_{1}^{*}$ [and hence $\mathcal{E}(1, \mu)$ ] were independent of $\mu, n(1)$ would still be nonlocal because of the denominator in Eq. (23).

One might at first wonder whether this nonlocal behavior is in some sense illusory, since it disappears when $N$ is eliminated in favor of $\mu$. Upon reflection, however, it becomes clear that this behavior is real and physical. The point is that in a closed system, any variations in $\mathbf{E}_{L}(\mathbf{r})$ must occur strictly at fixed $N$. Variations at fixed $\mu$ are unphysical in such a system, since they are accompanied by a variation in $N$. (They are still useful mathematically, however, as our development illustrates.) Variations at fixed $N$ are most easily contemplated by eliminating $\mu$ in favor of $N$. Equation (23) then shows clearly that even if $\mathbf{E}_{L}(\mathbf{r})$ varies only at points far from $\mathbf{r}_{1}$, the value of $n(1)$ will change, i.e., $n(1)$ is affected by what happens to $\mathbf{E}_{L}(\mathbf{r})$ at points far from $r_{1}$, which is just what is meant by nonlocal behavior.

Our conclusion that $n(1)$ in a closed system is inherently nonlocal in $\mathbf{E}_{L}(\mathbf{r})$ is in conspicuous disagr eement with the result of Nienhuis and Deutch, ${ }^{11}$ who obtained a formal expression for $n(1)$ that is purely local in $E_{L}\left(r_{1}\right)$ for slowly varying fields [their Eq. (4.30)]. It seems likely that this disagreement is due to the fact ${ }^{12}$ that ND neglect the finite-volume correction terms that appear in the correlation functions of a finite system in the canonical ensemble. ${ }^{17}$ As we have shown elsewhere, ${ }^{13}$ these terms profoundly alter the usual functional-derivative relations, and their omission will in general lead to serious error. The finite-volume correction to $h(12)$ has the effect of replacing $\delta \gamma_{0}(1)$ by $\delta \gamma_{0}(1)+\beta \delta \mu$ in Eq. (2), with $\delta \mu=(\partial / \partial N) \int d(1) n(1) \delta \phi_{0}(1)$. It is through the nonlocal quantity $\delta \mu$ that nonlocal behavior manifests itself in the canonical ensemble. In the linear approximation, however, the finite-volume correction terms have no effect, because $n(1)$ is then replaced by $\bar{\rho} / \Omega$ in the expression for $\delta \mu$, and $\delta \mu$ then vanishes by virtue of $\mathrm{Eq}$. (1) (since $\int d \boldsymbol{\omega}_{1} \mathrm{~m}_{1}=0$ ). Cons equently, $n(1)$ is a local function of $\mathbf{E}_{L}\left(\mathbf{r}_{1}\right)$ in the linear approximation but not in higher order, as we now proceed to show directly.

In the linear approximation, $\mathcal{E}(1, \mu)$ becomes

$$
\mathcal{E}(1, \mu)=1+\beta \mathbf{m}_{1}^{*}(1,0) \cdot \mathbf{E}_{L}\left(\mathbf{r}_{1}\right),
$$

where $m_{1}^{*}(1,0)$ still depends implicitly on $\mu$. In zero field, however, it is reasonable to assume that the short-ranged function $h_{0}(12)$ depends only on the relative positions and orientations of molecules 1 and 2 . This implies that $\mathbf{m}_{1}^{*}(1,0)$ is a vector rigidly affixed to molecule 1 , so that $\int d \omega_{1} m_{1}^{*}(1,0)=0$ and $\int d(1) \mathcal{E}(1, \mu)=V \Omega$. Equation (22) then reduces to $N=V \rho_{0}(\mu, T)$, which no longer involves $\mathbf{E}_{L}(\mathbf{r})$. In the linear approximation, therefore, $\mu$ is no longer a functional of $\mathbf{E}_{L}(\mathbf{r})$; indeed, it is just the chemical potential $\mu_{0}$ of the unperturbed $N$ molecule system. Equation (23) for $n(1)$ now becomes

$$
n(1)=(N / V \Omega)\left[1+\beta \mathbf{m}_{1}^{*}(1,0) \cdot \mathbf{E}_{L}\left(\mathbf{r}_{1}\right)\right]
$$

where $m_{1}^{*}(1,0)$ is to be evaluated at $\mu=\mu_{0}$. Equation (25) shows that $n(1)$ is a purely local function of $\mathbf{E}_{L}\left(\mathbf{r}_{1}\right)$ in the linear approximation. It is clear, however, that this simple local behavior no longer obtains in quadratic and higher order.

It is of interest to examine the local number density $\rho\left(\mathbf{r}_{1}\right)=\int d \omega_{1} n(1)$, which according to $\mathrm{Eq}$. (23) is given by

$$
\rho\left(\mathbf{r}_{1}\right)=\frac{N \int d \boldsymbol{\omega}_{1} \mathcal{E}(1, \mu)}{\int d(1) \mathcal{E}(1, \mu)} .
$$


Equation (24) shows that in the linear approximation, $\rho(\mathbf{r})$ is uniform with the value $\bar{\rho}$. In higher order, however, it is clear that $\rho(\mathbf{r})$ is a nonlocal functional of $\mathbf{E}_{L}(\mathbf{r})$ and is no longer uniform. The fact that $\rho(\mathbf{r})$ becomes nonuniform in a strong electric field has also been observed by Hoye and Stell. ${ }^{9}$ The physical reason for this behavior is straightforward. The primary effect of the electric field is to preferentially align the dipolar molecules. Once they are aligned, they will experience a nonzero net force due to the field gradient. This force will then redistribute the dipoles spatially, so that in equilibrium a nonuniform $\rho(\mathbf{r})$ will result. This physical picture makes it intuitively clear why $\rho(\mathbf{r})-\bar{\rho}$ is of second order in the field.

Equation (26) allows $n(1)$ to be written in the alternative form

$$
n(1)=\frac{\rho\left(\mathbf{r}_{1}\right) \mathcal{E}(1, \mu)}{\int d \omega_{1} \mathcal{E}(1, \mu)} .
$$

If $\mathrm{m}_{1}^{*}$ were independent of $\mu$, then $\mathcal{E}(1, \mu)$ would also be independent of $\mu$ and hence would be a purely local func tion of $\mathbf{E}_{L}\left(\mathbf{r}_{1}\right)$. [According to Eq. (22), however, $\mu$ itself would remain a nonlocal functional of $\mathbf{E}_{L}(\mathbf{r})$.] Equation (27) shows that in this case, $n(1)$ is the product of $\rho\left(\mathbf{r}_{1}\right)$ and a purely local function of $\mathbf{E}_{L}\left(\mathbf{r}_{1}\right)$, so that the nonlocal behavior of $n(1)$ is entirely contained in $\rho\left(\mathbf{r}_{1}\right)$. Moreover, the nonlocal behavior of $\rho(\mathbf{r})$ then becomes much simpler in character, since the $\mu$ dependence disappears from Eq. (26). Of course, this simple situation will never rigorously obtain, but it may obtain to a us eful degree of approximation under some circumstances.

\section{CONCLUDING REMARKS}

We have obtained a new formal expression for the single-molecule distribution function $n(1)$ in a finite dipolar fluid system. The us efulness of this expression depends upon one's ability to devise a satisfactory approximation to the effective moment $\mathbf{m}_{1}^{*}$. Since $\mathbf{m}_{1}^{*}$ has an appealing physical interpretation as the dipole moment of a deformable quasiparticle, it is perhaps not unreasonable to hope that simple physically motivated approximations to its behavior may be useful.

Our result shows that $n(1)$ is an inherently nonlocal functional of $\mathbf{E}_{L}(\mathbf{r})$ except in the linear approximation. To our knowledge, this nonlocal behavior has not previously been discussed, and it may at first appear somewhat surprising, since we have grown accustomed to local behavior from the linear regime. However, the necessity for nonlocal behavior is already clear in the case of an ideal gas. Our result may be specialized to this case by setting $\mathbf{m}_{1}^{*}=\mathbf{m}_{1}$, and noting that $\mathbf{E}_{L}(\mathbf{r})$ now differs negligibly from $\mathbf{E}_{0}(\mathbf{r})$. Equations (21) and (23) then immediately reduce to the correct $n(1)$ for an ideal gas, which is still inherently nonlocal in character.

The appearance in our theory of the Lorentz field $\mathbf{E}_{L}(\mathbf{r})$ rather than the Maxwell field $\mathbf{E}(\mathbf{r})$ is simply a consequence of our use of the tensor $T_{\delta}(r)$ to define $\theta(12)$. If we had instead defined $\theta(12)$ using the tensor ${ }^{6} \mathbf{T}_{\mathrm{ND}}(\mathbf{r})$, the development would have proceeded in formally identical fashion, but with $\mathbf{E}(\mathbf{r})$ replacing $\mathbf{E}_{L}(\mathbf{r})$. Of course, if this were done, then $c_{0}(12), h_{0}(12)$, and $\mathrm{m}_{1}^{*}$ would be different from the quantities that appear in the present development. One would then find that $n(1)$ is an inherently nonlocal functional of $\mathbf{E}(\mathbf{r})$. This is also clear in the present development, since $\mathbf{E}(\mathbf{r})=\mathbf{E}_{L}(\mathbf{r})-(4 \pi /$ 3) $\mathbf{P}(\mathbf{r})$, and $\mathbf{P}\left(\mathbf{r}_{1}\right)$ depends locally on $n(1)$.

The implications of the present results for the polarization $\mathbf{P}(\mathbf{r})$ and higher angular moments of $n(1)$ will be explored in a subsequent article. However, it is already obvious by inspection of Eqs. (11) and (23) that $\mathbf{P}(\mathbf{r})$ is in general an inherently nonlocal functional of $\mathbf{E}_{L}(\mathbf{r})$ or $\mathbf{E}(\mathbf{r})$ [although it becomes local in the linear regime because of Eq. (25)]. The statement to the contrary by Nienhuis and Deutch, ${ }^{11}$ embodied in their Eqs. (4.32) and (4.34), is ther efore erroneous. [This statement and these equations were based on their Eq. (4.30), which is incorrect for the reasons already given.] However, when $m_{1}^{*}$ is independent of $\mu$ the nonlocal behavior of $\mathbf{P}(\mathbf{r})$ becomes much simpler in character. In this case $\mathbf{P}(\mathbf{r})$, like $n(1)$ itself, depends nonlocally on $\mathbf{E}_{L}(\mathbf{r})$ only through $\rho(\mathbf{r})$, and one finds that the ratio $\mathbf{P}(\mathbf{r}) / \rho(\mathbf{r})$ (which is just the mean dipole moment per molecule at the point $\mathbf{r}$ ) is a purely local function of $\mathbf{E}_{L}(\mathbf{r})$. Even in the general case, the quantity $\mathbf{P}(\mathbf{r}) / \rho(\mathbf{r})$ should be simpler and more fundamental than $\mathbf{P}(\mathbf{r})$ itself whenever $\rho(\mathbf{r})$ is nonuniform.

Finally, it is of inter est to observe that the nonlocal behavior discussed in this article is of a very general type that is not intrinsically connected with the longrange nature of the dipolar force. Consequently, it differs in character from the more familiar nonlocal effects in dielectric theory, such as the nonlocal relation between $\mathbf{P}(\mathbf{r})$ and $\mathbf{E}_{0}(\mathbf{r})$ in the linear regime. ${ }^{1}$ It is instructive to contemplate how the development of Sec. II would be altered in the case of purely short-ranged intermolecular interactions. There is then no separation of $c$ (12) into short - and long-ranged parts; this separation may be negated by setting $\theta(12)=0$, so that $c_{0}(12)=c(12)$, $h_{0}(12)=h(12)$, and $\mathbf{E}_{L}(\mathbf{r})=\mathbf{E}_{0}(\mathbf{r})$. The entire derivation remains valid under these conditions, although many of the steps in it become trivally unnecessary. Consequently, one finds that $n(1)$ is a purely local function of $\mathbf{E}_{0}\left(\mathbf{r}_{1}\right)$ at constant $\mu$, but that nonlocal behavior appears just as before when $\mu$ is eliminated in favor of $N$. It is clear that this type of nonlocal behavior will be a general feature of strongly perturbed systems, regardless of the range of the inter molecular forces. It may therefore be of interest in other contexts.

\section{ACKNOWLEDGMENT}

I am grateful to George Stell for stimulating me to reexamine dielectric saturation, for many helpful comments and suggestions, and for providing me with a copy of Ref. 9 prior to publication.

\footnotetext{
1J. D. Ramshaw, J. Chem. Phys. 55, 1763 (1971).

2J. D. Ramshaw, J. Chem. Phys. 57, 2684 (1972).

${ }^{3} \mathrm{~J}$. D. Ramshaw, J. Chem. Phys. 64, 3666 (1976).

${ }^{4} \mathrm{~J}$. D. Ramshaw, J. Chem. Phys. 66, 3134 (1977).

${ }^{5}$ J. D. Ramshaw, J. Chem. Phys. 68, 5199 (1978).
} 
${ }^{6}$ J. D. Ramshaw, J. Chem. Phys. 70, 1577 (1979).

${ }^{7} \mathrm{~J}$. K. Percus, Phys. Rev. Lett. 8, 462 (1962).

${ }^{8}$ G. S. Rushbrooke, in Physics of Simple Liquids, edited by H. N. V. Temperley, J. S. Rowlinson, and G. S. Rushbrooke (Wiley-Interscience, New York, 1968), p. 25.

${ }^{9} \mathrm{~J}$. S. Hoye and G. Stell, J. Chem. Phys. 72, 1597 (1980).

${ }^{10} \mathrm{~S}$. Kielich, in Dielectric and Related Molecular Processes, edited by M. Davies (Chemical Society, London, 1972), Vol. 1 , p. 192 .

${ }^{11} \mathrm{G}$. Nienhuis and J. M. Deutch, J. Chem. Phys, 56, 1819 (1972).

${ }^{12}$ G. Nienhuis and J. M. Deutch, J. Chem. Phys. 55, 4213
(1971).

${ }^{13} \mathrm{~J}$. D. Ramshaw, "Functional-derivative relations for a finite nonuniform molecular fluid in the canonical ensemble," Mol.

Phys. (to be published).

${ }^{14}$ A. R. Edmonds, Angular Momentum in Quantum Mechanics (Princeton University, Princeton, 1960).

${ }^{15}$ A. D. Buckingham and J. A. Pople, Proc. Phys. Soc. London Sect. A 68, 905 (1955).

${ }^{16} \mathrm{~J}$. D. Ramshaw, Ph. D. thesis, Massachusetts Institute of Technology (1970), Chap. 3 .

${ }^{17}$ J. L. Lebowitz and J. K. Percus, Phys. Rev. 122, 1675 (1961). 